\title{
Opium Consumption Prevalence Among Patients With Ischemic Stroke Compared With Healthy Individuals in Iran
}

\author{
Alia Saberi, ${ }^{1}$ Amir Reza Ghayeghran, ${ }^{1}$ Sara Janeshin, ${ }^{2}$ Glareh Biazar, ${ }^{3,}$ and Ehsan Kazemnezhad ${ }^{4}$ \\ ${ }^{1}$ Neuroscience Research Center, Department of Neurology, Poursina Hospital, School of Medicine, Guilan University of Medical Sciences, Rasht, Iran \\ ${ }^{2}$ Faculty of Medicine, Guilan University of Medical Sciences, Rasht, Guilan, IR Iran \\ ${ }^{3}$ Anesthesiology Department, Anesthesiology Research Center, Guilan University of Medical Sciences, Rasht, Guilan, IR Iran \\ ${ }^{4}$ Biostatistics Department, Faculty of Nursery, Guilan University of Medical Sciences, Rasht, Guilan, IR Iran \\ "Corresponding author: Gelarh Biazar, Anesthesiology Department, Anesthesiology Research Center, Guilan University of Medical Sciences, Rasht, Guilan, IR Iran. Tel: \\ +98-9111302487, Fax: +98-13323337346, E-mail: golbiazar@gmail.com
}

Received 2015 January 24; Revised 2015 December 02; Accepted 2016 February 07.

\begin{abstract}
Background: One of the supposed risk factors of ischemic stroke is opium addiction, while the researches about its prevalence among patients with stroke are limited.

Objectives: The aim of this study was comparing the prevalence of opium addiction between subjects with thrombotic ischemic stroke and healthy individuals.

Patients and Methods: In this comparative cross-sectional study carried out in a number of academic hospitals affiliated to Guilan University of Medical Science in the north of Iran from April 2013 to March 2014, 83 patients involved by ischemic stroke and 83 control subjects contributed. Both groups were selected by simple sampling method. We tried to match the groups in terms of age, gender and socioeconomic condition during sampling. Those were assessed from the aspects of demographic data, risk factors of stroke, opium dependence, dosage and the methods of consumption, and the data was recorded in a designed checklist. The data were analyzed using chi-squared test, independent t-test, and multiple logistic regression models in SPSS software version 19 . P $<$ 0.05 was considered as the level of significance.

Results: A total of 83 patients (47\% female and 53\% male) with mean age of $68.55 \pm 15.01$ years and 83 control subjects ( $37.3 \%$ female and $62.7 \%$ male) with mean age of $67.94 \pm 13.55$ years $(P=0.782)$ were assessed; $39.8 \%$ of the case group and $20.5 \%$ of the control group consumed opium with no significant difference $(\mathrm{P}=0.007)$. There was no difference in the amount and methods of opium consumption between the two groups. After the adjustment of the effects of underlying and confounding variables, opium was not determined as an associated factor with stroke $(\mathrm{OR}=1.95, \mathrm{P}=0.096)$.

Conclusions: Opium was not identified as a risk factor of ischemic stroke. A more extended survey considering further confounding factors is recommended.
\end{abstract}

Keywords: Stroke, Ischemia, Opium, Dependence

\section{Background}

Drug abuse is one of the important general health problems in most countries, especially in Middle East, as well as in recent decades in Iran, and may be responsible for the ascending slope of mortality rate worldwide. It is also a considerable cause of disorders worldwide $(1,2)$. Around the world, there are 13.5 million opium-addicted people (3) and the prevalence of opium addiction in Iran during the past 20 years has been estimated to be increased nearly three folds, i.e. $2 \%-2.8 \%$ (4). The total incidence of its related disorders that are recorded in primary health centers have been estimated as $7 \%-8 \%$ (1).

Cerebral stroke is the third culprit of mortality and morbidity in the world and is the most common in neurology departments $(5,6)$. The stroke prevalence is calculated in developed countries as 100-300 in 100000 individuals per year and in Iran as 372 in 100000 per year (7). From 20 years ago, the financial expenses of cerebral stroke have increased by $76 \%$ (the prevalence statistics was $45 \%$ ), because of the increase of hospitalization days (8).

Cerebrovascular events are the sixth cause of disorders burden worldwide and are expected to reach to fourth in ranking (9).

More than $80 \%$ of stroke mortality in the world occurs in developing countries (10). In spite of these facts, it is preventable to some extent; so, identification and awareness of its risk factors in a particular country are the essential steps toward decreasing the stroke prevalence and its burden (4); one of the suspicious risk factors in Iran is opium consumption (5).

Nowadays, drug abuse is considered as a risk factor of stroke, especially in youngsters and adolescents. The considerable mechanisms are endocarditis with associated cerebrovascular damage, direct toxicity impact on vessels, 
substance embolization, changed vessels function, and immune-dependent injury to vessels, also referred as vasculitis (11). Of course, most of the above mentioned mechanisms are suggested only for intravenous drug abuse and fortunately not for which is discussed in this essay.

Opium is derived from poppy, and in comparison with pure opioid substance, it is a compound of intergradients of more than 20 alkaloids $(2,12)$. Morphine is one of the principle particles in fortified alkaloid latex in poppy, whereas poppy is consisted of nearly 80 terahydrobenzil which affect human metabolism and endocrine system and therefore can probably be different from pure morphine (13).

Opium and morphine can induce the decrement of total oxygen consumption and increase the coagulation potential and serum level of fibrinogen, which can lead to peripheral and cerebral arterial thrombosis and consequently cerebral stroke (5). Morphine can inhibit the function of calcium and venous endothelial growth factor (VEGF) and increase the serum level of total cholesterol, so be effective on vasospasm and myocardial infarction (14, 15). On the other hand, there is a belief among Asian and Middle Eastern populations and even doctors that opium can be a substituted or adjunctive treatment for diabetes or hyperlipidemia and be another atherosclerosis risk factors. Of course in some studies, it is ineffective or even has the contrary effects (13, 16-18).

Stroke prevention is superior to its treatment; so, investigations are inclined to determine its risk factor, and by introducing it to the general population take steps to prevention of this disorder. Till now, not many reports about the association between opium addiction and thrombotic ischemic stroke have been conducted. Furthermore, in Guilan province in north of Iran, some farmers and older people consume opium to diminish their musculoskeletal pain, so the present study tried to survey the above mentioned coincidence.

\section{Objectives}

This study examined the association between opium addiction and risk of ischemic stroke in north of Iran.

\section{Patients and Methods}

The present comparative cross-sectional survey was carried out in two academic hospitals associated with Guilan University of Medical Science in north of Iran, from April 2013 to March 2014.

The sample size was accounted based on Hamzei Moghadan A. study (5) by confidence of 95\% and test power of $90 \%$ and the odds ratio of $0.372 \%$, using the following formula with the minimum set as 72 subjects in each group, since there was no study such as ours.

$$
\begin{aligned}
n= & \frac{\left(z_{1-\frac{\alpha}{2}}+z_{1-\beta}\right)^{2}}{P(1-P)(\text { Lnoddsratio })^{2}}=\frac{(1.96+1.28)^{2}}{0.372(1-0.372)(L n 2.207)^{2}}=71.772 \\
& \alpha=0.05 \\
& 1-\alpha=95 \% \\
& 1-\beta=90 \% \\
& P=0.372 \% \text { (the prevalence of stroke in Iran according }
\end{aligned}
$$
to reference 7 )

Odds ratio $=\mathbf{2 . 2 0 7}$

Considering approximately $10 \%$ dropout, eventually 83 subjects for each group were enrolled in the survey. Both groups were selected by simple sampling method. Entering into this survey depended to fulfilling the informed consent by the case and control subjects or their legal responsible. Totally 83 patients with ischemic stroke confirmed by clinical and CT and/or MRI findings comprised the case group. The control subjects group was made of 83 age-and-gender-matched patients admitted in urology wards because of the most similarities in age and culture and socioeconomic conditions to the case group and also the most probability of absence of the special background disorders different from patients with stroke. We tried to match groups as age and gender and socioeconomic conditions during sampling by individualized matching.

The patients who were referred to the determined hospital had the same socioeconomic conditions. The exclusion criteria were: history of drug usage which accelerate ischemic stroke including vasopressors and estrogencontaining compound, past history of cerebral stroke, head trauma, venous infarction, metabolic disorders including uremia, abnormal liver function test more than three times as normal values, cardiac embolic source confirmed by echocardiography, suspicious metastatic or other space occupying lesion, and less than one year of opium addiction and impossibility to give acceptable history. Opium addiction was defined according to DSM-IV-TR (304.00, opium dependence).

The data were entered in a designed checklist including social and past medical history, physical and neurological exam, imaging findings, demographic information, opium addiction, the amount of opium used (which was relatively clear for consumers because of its high price and obsession in its usage), the route of consumption and the data of withdrawal if there was, and also the type of involved vessels (lacunar or large vessel). All subjects had been assured about their data being confidential. 


\section{Results}

A total of 166 subjects contributed in the present survey. Eighty three patients with ischemic stroke ( $47 \%$ females and $53 \%$ males) with a mean age of $68.55 \pm 15.01$ years formed the case group and the others (37.3\% females and $62.7 \%$ males) with a mean age of $67.94 \pm 13.55$ years made up the control subjects. No statistically significant difference in terms of gender and age was found between the two groups ( $\mathrm{P}=0.209$ and 0.782 , respectively). They were significantly different in terms of some stroke risk factors including hypertension $(\mathrm{P}=0.001)$, smoking $(\mathrm{P}=0.018)$ and family history of stroke $(\mathrm{P}=0.006)$, but there was no significant difference in diabetes mellitus $(\mathrm{P}=0.518)$ and dyslipidemia $(\mathrm{P}=0.074)$ (Table 1 , chi-squared test).

Among the case group, the prevalence of opium usage was 39.8\% $(\mathrm{n}=33)$ and in the control group it was 20.5 $(\mathrm{n}=17)$ without any statistically significant difference $(P$ $=0.007)$. The path and amounts of opium consumption were stated by 32 patients (from 33) and 12 control subjects (from 17); there was no marked difference in the manner of usage ( $\mathrm{P}=0.999$, Fisher's exact test) (Table 2 ).

Regarding the mean amount of opium consumption (g/day), there was no significant difference between them ( $0.84 \pm 0.34$ vs. $0.71 \pm 0.33 \mathrm{~g} /$ day, $\mathrm{P}=0.266$, t-test).

The mean duration of opium consumption was stated by all the cases and by only 10 control subjects, which were estimated $131.6 \pm 120$ and $72.0 \pm 44.2$ months, respectively. It was clear that a very significant difference existed $(\mathrm{P}=$ 0.024 , t-test) and patients with stroke consumed opium longer than the controls as means of 59.06 months (95\% CI: 8.1-109.98).

To control the effect of other confounding or background factors, backward logistic regression(LR) stepwiselogistic regression model was used. All of the parameters with P values less than 0.25 determined in uni-variate analysis were entered in this model. The final model of multivariate logistic regression with probability of entering and existing of 0.05 and 0.1 is summarized in Table 3. Based on this model, opium was also not determined as a risk factor of ischemic stroke $(\mathrm{P}=0.096)$.

However, as mentioned in the above table, the positive history of smoking also remained as a significant predictor $(\mathrm{P}=0.029, \mathrm{OR}=2.2,95 \% \mathrm{CI}: 1.087-4.809)$ such as hypertension $(\mathrm{P}=0.001, \mathrm{OR}=3.39,95 \% \mathrm{CI}: 1.639-7.01)$.

In the assessment of the relationship between involved vessels and opium, we could not find any significant results ( $P>0.05)$, so that $40.19 \%$ of cases had lacunar infarct and $41 \%$ had large vessels.

\section{Discussion}

The present study showed that the prevalence of opium usage among the case group was not significantly different from the control subjects. In the backward LR stepwise-logistic regression model, opium also was not determined as a risk factor of ischemic stroke.

In addition, there was not significant difference in the manner and the mean amount of opium consumption between the groups. The mean duration of opium consumption was stated by all the cases and by only 10 control subjects and we achieved a very significant difference in the appearance, so that patients with stroke consumed opium longer than the controls as many as approximately 60 months.

In the assessment of the relationship between involved vessels and opium, we could not find any significant difference between small vessels and large vessels involvement.

In some cardiovascular studies such as Mohammadi's and Sadighian's, opium was introduced a promoter of atherosclerosis $(15,18)$, but Shirani et al. demonstrated that opium was not a cardiovascular protector (2). In another survey around this subject, Asgari also showed that opium did not have any impression on lipid profile and the serum level of glucose (16).

Considering the results of cardiovascular studies, the effect of opium on stroke was also supposed to be assessed. Hamzeimoghadam marked this relation in his study, during which around $29.5 \%$ of patients with stroke were accounted to be opium addicted, which was three times more than the control subjects calculated as $10.50 \%$ ( $\mathrm{P}<$ 0.001 ) (5). In the present study, although there was a $19 \%$ difference between patients with stroke and controls regarding the prevalence of opium usage, the difference was not statistically significant, which is in conflict with the last study by Hamzei-Moghaddam. In the context of another survey by Hamzeimoghadam in 2013, it was concluded that the pattern of great vessels stenosis was not associated with opium addiction (19). The final result of our study also concluded that the involvement of large or small vessel was not affected by opium, similar to this scholar study (19). There are some evidences which declare that opium can increase the serum level of fibrinogen and coagulation potential and atherosclerosis by affecting the lipid profile $(17,18,20,21)$. Nevertheless these concepts were present, it seemed that they were not strong enough to make opium as a high potential risk factor for cerebral stroke as cardiovascular involvement. Therefore, there are such varieties in the results of different studies and they could not achieve a unique conclusion. Interestingly, one of the popular conceptions is that opium can lessen the serum level of blood glucose and lipids as stroke risk fac- 
Table 1. Comparison of the Distribution of Gender and Stroke Risk Factors Between Case and Control Groups

\begin{tabular}{|c|c|c|c|c|}
\hline & \multicolumn{4}{|c|}{ Studied Groups } \\
\hline & Case, No. (\%) & Control, No. (\%) & Total, No. (\%) & P.Value \\
\hline Gender & & & & 0.209 \\
\hline Male & $44(53)$ & $52(62.7)$ & $96(57.8)$ & \\
\hline Female & $39(47)$ & $31(37.3)$ & $70(42.2)$ & \\
\hline Diabetes mellitus & $32(38.6)$ & $28(33.7)$ & $60(36.1)$ & 0.518 \\
\hline Dyslipidemia & $35(42.2)$ & $24(28.9)$ & $59(35.5)$ & 0.074 \\
\hline Arterial hypertension & $62(74.7)$ & $42(50.6)$ & $104(62.7)$ & 0.001 \\
\hline Smoking & $42(50.6)$ & $26(31.3)$ & $68(41.0)$ & 0.018 \\
\hline Positive family history of stroke & $32(38.6)$ & $16(19.3)$ & $48(28.9)$ & 0.006 \\
\hline
\end{tabular}

Table 2. Comparison of the Manner of Opium Usage

\begin{tabular}{|c|c|c|c|c|c|}
\hline & & \multicolumn{3}{|c|}{ The Manner of Opium Usage } & \multirow[t]{2}{*}{ Total } \\
\hline & & Inhalation & Oral & Inhalation + Oral & \\
\hline \multirow{2}{*}{ Groups } & Patients, No. (\%) & $5(15.6)$ & $26(81.3)$ & $1(3.1)$ & $32(100)$ \\
\hline & Controls, No. (\%) & $2(16.7)$ & $10(83.3)$ & $0(0)$ & $12(100)$ \\
\hline Total & No. (\%) & $7(15.9)$ & $36(81.1)$ & $1(2.3)$ & $44(100)$ \\
\hline
\end{tabular}

Table 3. The Final Model of the Association of Opium Addiction and Ischemic Stroke with Controlling the Confounding Factors

\begin{tabular}{|c|c|c|c|c|c|c|}
\hline & \multirow{2}{*}{$\begin{array}{c}\text { B (Regression } \\
\text { Coefficient) }\end{array}$} & \multirow{2}{*}{$\begin{array}{l}\text { Standard } \\
\text { Error }\end{array}$} & \multirow[t]{2}{*}{ P. Value } & \multirow[t]{2}{*}{ Odds Ratio } & \multicolumn{2}{|c|}{ 95\% CI for Odds Ratio } \\
\hline & & & & & Lower & Upper \\
\hline Positive history of opium addiction & 0.666 & 0.4 & 0.096 & 1.946 & 0.889 & 4.261 \\
\hline Positive history of smoking & 0.827 & 0.379 & 0.029 & 2.286 & 1.087 & 4.809 \\
\hline Positive history of hypertension & 1.221 & 0.371 & 0.001 & 3.39 & 1.639 & 7.01 \\
\hline Positive family history of ischemic stroke & 0.683 & 0.384 & 0.075 & 1.981 & 0.933 & 4.206 \\
\hline Gender (male) & 0.656 & 0.358 & 0.067 & 1.926 & 0.955 & 3.887 \\
\hline Constant & -2.451 & 0.673 & 0.000 & 0.086 & & \\
\hline
\end{tabular}

tors, but as it was previously pointed, this idea was also rejected (16).

Along with the above mentioned fact, it is necessary to declare that the investigators tried to match the two groups respecting age, gender and other stroke risk factors, but it was not possible for hypertension, smoking and family history of stroke. Hence, their effects were controlled by multivariate logistic regression model. Furthermore, according to this model as pointed, hypertension and smoking strongly remained as the risk factors of ischemic stroke which were proved in ancient studies and there is no doubt about their roles.

There was no association between the stroke arising and the manner of consumption including oral or inhalation, whereas from the view point of Rezvani et al., oral consumption of opium can be a protective way in overcoming stroke, while inhalation cannot be one (22). Asgari denoted that the oral route can increase the serum level of morphine more than the inhaled form (17). Of course, it is not clear which high or low serum level of morphine can be protective in facing stroke.

No association was also determined between stroke and the amount consumed opium. Nonetheless an association was found between stroke and duration of opium usage, as the patients with stroke used opium averagely 39 month longer than the control subjects, we cannot rely 
on this finding, because only 10 control subjects from the opium consumers declared the duration of opium consumption, whereas all 33 cases stated it. However, in case of being a reliable result, it can be explained for this finding that perhaps, either the increment of the serum level of morphine or the induction of tolerance are dependent to opium, and thus, gradual increase of the amount of opium usage is its result. Although the latter was not established as mentioned above, in confirmation of the former, Asgari et al. in the context of cardiovascular risk factors declared that long duration of opium addiction leads to higher serum level of morphine, which may have impact on stroke occurrence which itself is yet in doubt (17).

There were some limitations in data gathering, as patients or their responsible people avoided declaring the consumption of opium. By assuring them of data confidentiality, we could relatively overcome this obstacle.

Although near half of all the patients with stroke in this survey were opium addicted, opium was not determined as a stroke risk factor. A more extended survey considering the further numbers of confounding factors which would present the definite level of significance is recommended.

\section{Acknowledgments}

This study was extracted from the thesis of Sara Janeshin for the degree of MD with registry number of 1697. We appreciate Dr. Hojjat Janeshin because of his immense cooperation and also vice-chancellorship of the medical faculty, Guilan University of Medical Sciences.

\section{Footnote}

Authors' Contribution: Alia Saberi designed the proposal, gathered the data, and wrote the manuscript; Amir Reza Ghayeghran gathered the data; Sara Janeshin gathered the data; Gelarh Biazar wrote the manuscript and analyzed the data; Ehsan Kazemnezhad analyzed the data.

\section{References}

1. Neushotz LA, Fitzpatrick JJ. Improving substance abuse screening and intervention in a primary care clinic. Arch Psychiatr Nurs. 2008;22(2):78-86. doi: 10.1016/j.apnu.2007.04.004. [PubMed: 18346564].

2. Shirani S, Shakiba M, Soleymanzadeh M, Esfandbod M. Can opium abuse be a risk factor for carotid stenosis in patients who are candidates for coronary artery bypass grafting?. Cardiol J. 2010;17(3):254-8. [PubMed: 20535715].

3. Bafghi SMS, Rafiei M, Bahadorzadeh L, Namayeh SM, Soltani MH, Andishmand MMA. Is opium addiction a risk factor for acute myocardial infarction?. Acta Medica Iranica. 2005;43(3):218-22.
4. Hosseini AA, Sobhani-Rad D, Ghandehari K, Benamer HT. Frequency and clinical patterns of stroke in Iran - Systematic and critical review. BMC Neurol. 2010;10:72. doi: 10.1186/1471-2377-10-72. [PubMed: 20731823].

5. Hamzei Moqaddam A, Ahmadi Musavi SM, Khademizadeh K. Relationship of opium dependency and stroke. Addict Health. 2009;1(1):6-10. [PubMed: 24494076].

6. Sarti C, Rastenyte D, Cepaitis Z, Tuomilehto J. International trends in mortality from stroke, 1968 to 1994. Stroke. 2000;31(7):1588-601. [PubMed: 10884459].

7. Dalvandi A, Ekman S-L, Maddah SSB, Khankeh HR, Heikkila K. Post Stroke life in Iranian people: used and recommended strategies. Iran Rehabi J. 2009;7(9):17-24.

8. Terent A, Marke LA, Asplund K, Norrving B, Jonsson E, Wester PO. Costs of stroke in Sweden. A national perspective. Stroke. 1994;25(12):23639. [PubMed: 7974574].

9. Menken M, Munsat TL, Toole JF. The global burden of disease study: implications for neurology. Arch Neurol. 2000;57(3):418-20. [PubMed: 10714674].

10. Lopez AD, Mathers CD, Ezzati M, Jamison DT, Murray CJ. Global and regional burden of disease and risk factors, 2001: systematic analysis of population health data. Lancet. 2006;367(9524):1747-57. doi: 10.1016/S0140-6736(06)68770-9. [PubMed: 16731270].

11. Caplan LR, Hier DB, Banks G. Current concepts of cerebrovascular disease-stroke: stroke and drug abuse. Stroke. 1982;13(6):869-72. [PubMed: 6755815].

12. J.S. . Drug abuse and dependence Cecil textbook of medicine. 2000

13. Karam GA, Reisi M, Kaseb AA, Khaksari M, Mohammadi A, Mahmoodi $M$. Effects of opium addiction on some serum factors in addicts with non-insulin-dependent diabetes mellitus. Addict Biol. 2004;9(1):53-8. doi: 10.1080/13556210410001674095. [PubMed:15203439].

14. Roy S, Balasubramanian S, Wang J, Chandrashekhar Y, Charboneau R, Barke R. Morphine inhibits VEGF expression in myocardial ischemia. Surgery. 2003;134(2):336-44. doi: 10.1067/msy.2003.247. [PubMed: 12947338].

15. Sadeghian S, Darvish S, Davoodi G, Salarifar M, Mahmoodian $\mathrm{M}$, Fallah $\mathrm{N}$, et al. The association of opium with coronary artery disease. Eur J Cardiovasc Prev Rehabil. 2007;14(5):715-7. doi: 10.1097/HJR.ob013e328045c4e9. [PubMed: 17925633].

16. Asgary S, Naderi GH, Soghraty M, Ahmady P, Shahrezaee JR. A study of plasma lipid peroxidation, lipids and blood sugar level in opium addicts compared with control group. ARYA Atheroscler. 2010;1(2).

17. Asgary S, Sarrafzadegan N, Naderi GA, Rozbehani R. Effect of opium addiction on new and traditional cardiovascular risk factors: do duration of addiction and route of administration matter?. Lipids Health Dis. 2008;7:42. doi:10.1186/1476-511X-7-42. [PubMed:18980684].

18. Mohammadi A, Darabi M, Nasry M, Saabet-Jahromi MJ, Malek-PourAfshar R, Sheibani H. Effect of opium addiction on lipid profile and atherosclerosis formation in hypercholesterolemic rabbits. Exp Toxicol Pathol. 2009;61(2):145-9. doi: 10.1016/j.etp.2008.08.001. [PubMed: 18838257].

19. Hamzei-Moghaddam A, Shafa MA, Khanjani N, Farahat R. Frequency of Opium Addiction in Patients with Ischemic Stroke and Comparing their Cerebrovascular Doppler Ultrasound Changes to Non-Addicts. Addict Health. 2013;5(3-4):95-101. [PubMed: 24494165].

20. Masoomi M, Nasri H, Farajpour F. Comparison of plasma Fibrinogen level in Opium addict men with non-addict men.J Kerman Univ Med Sci. 2002;9(1):27-31.

21. Baird TA, Parsons MW, Barber PA, Butcher KS, Desmond PM, Tress $\mathrm{BM}$, et al. The influence of diabetes mellitus and hyperglycaemia on stroke incidence and outcome. J Clin Neurosci. 2002;9(6):618-26. [PubMed: 12604269].

22. Rezvani MR, Ghandehari K. Is opium addiction a risk factor for ischemic heart disease and ischemic stroke?. J Res Med Sci. 2012;17(10):958. 